Meta

Journal des tradlucteurs

Translators' Journal

\title{
Les voix plurielles de la traduction de Camus en hébreu
}

\section{Cyril Aslanov}

Volume 44, numéro 3, septembre 1999

URI : https://id.erudit.org/iderudit/001910ar

DOI : https://doi.org/10.7202/001910ar

Aller au sommaire du numéro

Éditeur(s)

Les Presses de l'Université de Montréal

ISSN

0026-0452 (imprimé)

1492-1421 (numérique)

Découvrir la revue

Citer cet article

Aslanov, C. (1999). Les voix plurielles de la traduction de Camus en hébreu. Meta, 44(3), 448-468. https://doi.org/10.7202/001910ar d'utilisation que vous pouvez consulter en ligne.

https://apropos.erudit.org/fr/usagers/politique-dutilisation/ 


\title{
Les voix plurielles de la traduction de Camus en hébreu
}

\author{
cyril aslanov \\ Université hébraïque de Jérusalem \\ Département de langue et littérature française
}

\begin{abstract}
RÉSUMÉ
Albert Camus, de part la richesse tant littéraire que philosophique de son œuvre, est un des auteurs les plus connus en Israël. Partant des nombreuses traductions faites de l'œuvre de Camus en hébreu, l'article analyse les différentes tendances de la traduction en Israël. Selon les options méthodologiques des traducteurs et suivant la nature du texte source, les tendances vont de la traduction communicative à la traduction informative et sémantique. Par ailleurs, les traduction oscillent principalement entre la littéralité et l'adaptation, avec un éventail d'autres genres de traductions entre les deux 〈voix» extrêmes.
\end{abstract}

\begin{abstract}
Albert Camus's literary and philosophical richness makes him one of the best known authors in Israel. Basing its approach on numerous Hebrew translations of Gmus's works, this article analyses different approaches of translation in Israel. Tanslators' methodological options and the type of the original text bring these approaches from communicative to informative and semantic translation. Fanslations fluctuate between literality and adaptation, with many other types of translations between two extreme "voices".
\end{abstract}

Entre les premières traductions d'Albert Camus en hébreu et celles qui sont parues tout récemment (je pense notamment à celles du Premier homme et de L'envers et l'endroit), la langue hébraïque a subi des transformations profondes. Dans les œuvres littérai res originales des écrivains jeunes ou moins jeunes, on constate une réduction très nette de l'écart entre langue parlée et langue écrite. En moins de cinquante ans, on est passé d'un état de quasi diglossie faisant coexister une langue parlée évoluant à toute allure avec une langue écrite figée dans un certain conservatisme, à une situation où les barrières entre les deux niveaux de langue n'ont plus rien d'infranchissable.

Cette évolution dans les habitudes d'écriture n'a pas manqué de se répercuter sur le style des traductions et en particulier sur les diverses traductions qui ont été effectuées à partir de l'œuvre de Camus. Pour bien apprécier l'ampleur de ces changements, nous avons choisi de comparer la traduction quelque peu surannée de La peste par le poète Yonathan Ratosh et d'autres traductions un peu plus modernes, comme celle de La chute, de L'exil et le royaume (1959) ou du M ythe de Sisyphe (1978) par Zvi Arad, celle de La mort heureuse par Aaron Amir (1980), ou nettement plus modernes, comme celle de L'étranger par Ilana Habermann (1985), celle de $N$ oces et L'été par Aviva Barak (1988) et enfin celle de L'envers et l'endroit par Adina Kaplan.

Cette confrontation des anciens et des modernes est certes importante du point de vue de I'histoire de la réception de l'œuvre de Camus en Israël. De fait, le traducteur porte une large part de responsabilité dans le succès qu'un auteur étranger peut 
rencontrer auprès d'un public alloglotte. M ais ce succès concerne surtout les destinées ultérieures de l'œuvre traduite. N ous voudrions tout d'abord concentrer notre attention sur le processus même de la traduction considérée d'un point de vue linguistique. Du fait même que Camus fut un auteur prolixe et que nombre de ses œuvres ont été traduites en hébreu par des traducteurs différents et à des périodes variées, la fortune littéraire de Camus en Israël constitue un excellent révélateur des tendances récentes ou moins récentes de la traduction en Israël.

Au-delà de ces considérations techniques qui intéressent surtout les traducteurs professionnels, la réflexion sur la version du texte de Camus en hébreu doit nous mener à la question de la conversion des contextes culturels, problématique incontournable de toute réflexion sur l'acte de traduction. Autrement dit, l'œuvre de Camus est-elle suffisamment universelle pour être comprise au prix d'une simple transposition langagière? Ou bien le passage d'une langue à l'autre doit-il s'accompagner dans le cas précis de cette œuvre d'une réinterprétation en termes accessibles au lecteur israélien de ce qui constitue l'ancrage spécifique de Camus dans sa culture d'origine, dans son époque et dans son Algérie natale? Pour cela, nous recourrons à une approche comparative qui fera intervenir des traductions dans d'autres langues (arabe, castillan, anglais, allemand).

\section{TRADUIRE LA LANGUE DE CAMUS: ENTRE ÉCRITURE BLANCHE ET LYRISME MÉDITERRANÉEN}

\subsection{La traduction de Yonathan Ratosh, analogon hébreu de l'écriture blanche?}

Dans Le degré zéro de l'écriture, Roland Barthes qualifie le style de Camus dans L'étranger d'«écriture blanche» et de "parole transparente», c'est-à-dire d'écriture dans laquelle la présence affective de l'écrivain et l'impact de ses représentations sociales et mythiques sont presque abolis (1972: 55-56). Techniquement parlant, cet effet est obtenu en français par l'absence complète d'emphase et par une sorte d'ascèse stylistique. Les phrases courtes comptant tout au plus une ou deux subordonnées l'emportent de loin sur les périodes chères à l'écriture dite bourgeoise.

Certes, La peste est écrite dans un style un peu moins minimaliste que L'étranger. On y trouve néanmoins l'annonce de ce dépouillement stylistique qui caractérise la voix intérieure de M eursault. En somme, l'épisode où Grand se trouve aux prises avec la première phrase de son roman Allées fleuries constitue la figure emblématique a contrario de l'idéal stylistique de Camus romancier : simplicité et naturel poussés à l'extrême. De la même façon, on trouve dans L'étranger un personnage dont le tic stylistique contraste délibérément avec la sobriété de Meursault, I'homme qui ne parle pas pour ne rien dire (II,3). II s'agit de M asson qui «avait I'habitude de complé ter tout ce qu'il avançait par un "et je dirai plus", même quand, au fond, il n'ajoutait rien à la phrase» $(I, 6)$. Signalons en outre que le concierge de l'asile de vieillards de $M$ arengo $(I, 1)$ se signale lui aussi par son verbiage incessant. À travers ces trois personnages un peu grotesques (et qui ont du reste en commun d'être des «Français de France», puisque Grand vient de M ontélimar et M asson et la concierge de Paris), il semble que Camus exprime par contraste son idéal stylistique tourné vers une sorte de dépouillement ascétique. 
Par un biais inattendu, le poète Yonathan Ratosh est parvenu à fournir un analogon de cette écriture blanche ou presque blanche de La peste. M ais au lieu de transposer en hébreu la sobriété extrême de l'écriture romanesque camusienne, il s'est efforcé de parvenir au même effet d'écriture impersonnelle en utilisant le truchement d'un style guindé, caractérisé par la prédilection pour les tours synthétiques (suffixes pronominaux, infinitifs construits et goût pour les constructions nominales). Car c'est un fait que l'idéal stylistique et la notion de style sublime varient selon les langues, fussent-elles apparentées. L'idéal de sobriété classique et de clarté limpide du français tel qu'il s'est élaboré au xvii ${ }^{e}$ siècle contraste avec la définition italienne ou espagnole de la belle prose, plus proche de la profusion asianique que de I'ascèse attique. Dans la perspective de la stylistique comparée, l'hébreu fait figure de langue dont l'idéal stylistique pourrait être qualifié d'ultra-attique: la préférence de la prose élégante pour les structures synthétiques et la concision remarquable du style biblique contrastent nettement avec les constructions analytiques de la langue parlée, laquelle est en outre infestée de xénismes syntaxiques provenant du yiddish, de l'allemand, du russe et plus récemment de l'américain. En sorte que le beau style hébreu et son homologue français (celui qui précède l'avènement de l'écriture bourgeoise aux boursouflures asianiques) ont peut-être plus de points communs qu'il n'y paraît au premier abord, le génie des deux langues tenant à un goût, plus ou moins marqué dans le cas du français et très net dans le cas de l'hébreu, pour la sobriété et la concision.

Quoi qu'il en soit, cette adéquation entre l'écriture blanche de Camus et la solennité de la prose de Ratosh traduisant Camus tient au fait que dans les deux cas le scripteur est étonnamment absent du texte qu'il produit. La chose a déjà été remarquée à propos du style de L'étranger et cette remarque vaut dans une large mesure pour La peste, même si le registre y est un peu plus élevé. Car enfin I'hébreu solennel de Ratosh n'est pas moins impersonnel que la voix blanche de Camus romancier. Ces artifices stylistiques répertoriés, souvent empruntés à l'hébreu biblique, sont des instruments de reconnaissance sociale entre lettrés, bien loin d'être des procédés emphatiques exprimant l'émotion du scripteur. II y a presque un détournement parodique de la solennité du style biblique. Derrière ces masques conventionnels, le traducteur israélien se dissimule avec autant sinon plus de discrétion que le romancier français ne le fait grâce à son style sobre et dépouillé. Car il est bien entendu qu'en dépit de son ancrage stylistique dans le registre biblique, la prose de Ratosh se distingue nettement de son verbe poétique «cananéen » où la phraséologie biblique est recyclée de façon créative et même subversive. Le style du traducteur Ratosh est un instrument de communication sociale, une sorte de classicisme un peu suranné.

Cette façon de transposer un style par un autre, différent mais équivalent mutatis mutandis, ressortit à la traduction dite communicative et dynamique, pour employer un concept de traductologie (voir Newmark 1972: 39). Toutefois, il est un point où cette adéquation de l'écriture solennelle hébraïque avec l'écriture blanche de Camus cesse de fonctionner. II s'agit des passages dialogués où Camus déploie ses qualités de dramaturge en prêtant à chacun le style qui lui convient le mieux. Même pour traduire ces répliques réalistes, voire même véristes, Yonathan Ratosh ne s'est pas départi de son style guindé. L'écart entre l'original et la traduction devient alors tellement flagrant qu'il en est parfois cocasse. Citons pêle-mêle les exemples suivants: 
Si tu crois que ça vaut vraiment la peine, je peux essayer d'obtenir un ordre.

אם אתה סבור שהדבר שוה באמת בטירחתו, אוכל לנסות לקבל צו.2

Dans l'original francais, l'emploi du pronom démonstratif ça au lieu de cela situe d'emblée cette phrase dans le registre de la conversation familière. En revanche, l'équivalent hébreu ressortit davantage au style guindé d'une lettre administrative: emploi du participe passif סבור à la place du participe présent חושב, du substantif

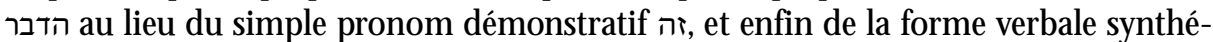
tique אוכל à l'imperfectif plutôt que du syntagme analytique combinant le pronom et le participe présent: אני יכול.

Citons un autre exemple allant dans le même sens. La phrase «Je l'ai décroché à temps», qui appartient au ton de la conversation la plus triviale, est pompeusement traduite par :

הורדתיו בעוד מועד.3

Citons encore la phrase «quand on est malade, il ne faut pas souffler dans un piston » rendue par le très formulaire

כשאדם חולה, אל לו לנשוף בחצוצרה.

On pourrait multiplier les exemples de ce décalage entre le style familier des parties dialoguées de l'original et le recours systématique au style pompeux dans la traduction. Cette étrange option de traduction obéit sans doute à un souci d'homogénéité stylistique au sein du texte-cible. Ayant jugé non sans raison que l'hébreu guindé convenait bien pour rendre la voix blanche du narrateur, Ratosh a étendu la recette là même où la voix blanche de l'original se fait pittoresque, voire même triviale ou vulgaire. M ais ce faisant, il a violél'équivalence communicative et dynamique qu'il avait lui-même établie entre la sobriété des parties narratives du textesource et le style guindé de la langue-cible.

\subsection{La traduction de L'étranger et de La mort heureuse par Aaron Amir : belles infidèles, faux sens et contresens}

Après avoir commis la première traduction de L'étranger en hébreu ${ }^{5}$, Aaron Amir $s^{\prime}$ attaqua au texte de La mort heureuse ${ }^{6}$. La traduction de L'étranger présente la particularité d'avoir pris pour base un Urtext du roman traduit en américain dès 19467. Cet ancrage de la première traduction israélienne dans un texte américain se manifeste notamment à travers l'emploi de la mesure de longueur מיל pour traduire les kilomètres du texte français: ainsi les «quatre-vingts kilomètres» qui séparent $\mathrm{Ma}$ arengo d'Alger et les «deux kilomètres» que Meursault parcourt entre ce dernier village et l'asile deviennent respectivement קצת יותר ממיל et כחמישים מיל Comme on le voit, l'acte de traduction nécessite parfois de procéder à des conversions numériques, en plus du passage de la langue-source à la langue-cible.

Contrairement à L'étranger, La mort heureuse semble avoir été traduit à partir de l'original français, comme l'attestent certains contresens qui ne peuvent s'expliquer qu'à partir de l'original.

Par rapport au style hiératique de Yonathan Ratosh, les traductions d'Aaron Amir se signalent par davantage de souplesse. N éanmoins il ne renonce pas au beau style tant qu'il s'agit de transposer la voix du narrateur. M ais les répliques des person- 
nages sont transposées dans un style vériste qui reflète l'argot israélien de l'époque. C'est dire que ce poète-traducteur a appliqué en l'affinant la méthode communicative et dynamique que son prédécesseur avait employée sans discernement. Le résultat est une belle traduction, pleine d'élégances, qui se laisse lire comme un texte original. $M$ ais comme il arrive souvent, le soin apporté au texte cible et le respect témoigné à l'égard de la langue-cible dissimulent une certaine désinvolture vis-à-vis de la languesource. On remarquera certaines maladresses dues à une connaissance insuffisante du français: c'est ainsi qu'au chapitre 2 de la première partie de La mort heureuse, il est question du «port gonflé de mâts et de grues noires». Et Aaron Amir de traduire par הנמל גדוש תרנים וזונות שחורות au terme d'une confusion entre le sens technique de grue et son sens argotique, lequel est dérivé du sens premier de ce mot, celui d'oiseau migrateur de la famille des échassiers. Le contresens est bien celui d'Aaron Amir, puisque «grues noires» a été traduit par black cranes dans la version américaine (Camus 1972).

On pourrait aussi mentionner au chapitre 2 de la deuxième partie un contresens dans la traduction de la proposition participiale «le cœur un peu barbouillé». Faute d'avoir identifiél'idiomatisme en vertu duquel le français situe par métonymie le mal de foie dans le cœur, Aaron Amir traduit ce syntagme par בלב נרגש קמעה. La première erreur qui consistait à traduire cet emploi figuré de cœur par לב a entraîné l'emploi non moins erroné de נרגש, participe renvoyant à un registre moral qui n'est pas de mise ici puisque Mersault est très prosaïquement victime d'une indigestion. Là encore, l'erreur prouve que contrairement à ce qui se produisit pour L'étranger, la traduction américaine n'a pas interféré. En effet, Richard Howard a très correctement et dynamiquement traduit «le cœur un peu barbouillé» par l'apposition feeling a little squeamish.

Au chapitre 2 de la première partie, l'image du «columbarium où des heures mortes auraient pourri » est escamotée, car Aaron Amir a pris le mot «columbarium » dans son acception étymologique de «pigeonnier», traduisant la phrase par שובך ששעות מתות מרקיבות בו. Là encore, nul soupçon d'interférence, puisque Richard Howard a préservé l'image macabre du texte original en rendant ce «columbarium » par catacomb. Le contresens ou du moins le faux sens d'Aaron Amir tient au fait que comme dans l'exemple ci-dessus, nous sommes en présence d'une similitude au second degré (il ne s'agit pas vraiment d'une métaphore, car le verbe évoquait qui introduit cette image a ici la fonction d'un expliciteur). Du point de vue collectif de la langue, une première métaphore assigne au mot latin signifiant «pigeonnier » le sens figuré et à vrai dire euphémique de «lieu où sont entreposées les urnes funéraires». Et du point de vue individuel du scripteur Camus, cette seconde acception dérivée sert à créer une image nouvelle en vertu d'une réactualisation de la métaphore heures mortes. Du reste cette similitude manque de cohérence, puisque dans un «columbarium» les cendres des défunts ne pourrissent pas. Quoi qu'il en soit, la traduction étymologisante de "columbarium » par montre qu'Aaron Amir en est resté au niveau du signifiant sans se rendre compte que le signifié rebondit ici à deux reprises, une première fois au terme d'une métaphore usée et une seconde fois en vertu d'une comparaison dûe à Camus.

Ces quelques exemples glanés çà et là dans la traduction de La mort heureuse révèlent la difficulté qu'a éprouvée le traducteur à faire la part des choses entre le sens premier et le sens dérivé de certains mots français: dansl'exemple des «grues noires», 
l'erreur ne tient pas tant au remplacement du sens propre par le sens figuré qu'à la confusion entre deux sens dérivés, celui de «machine servant à charger et à décharger les navires» et celui de «prostituée», tous deux se rattachant par des biais différents au signifié originel d'oiseau migrateur de la famille des échassier ${ }^{8}$. Dans le cas du «columbarium » et du «cœur barbouillé» au contraire, Aaron Amir, pourtant si à l'aise dans l'art des belles infidèles, a péché par excès de littéralisme en négligeant de rendre la véritable acception de ces termes.

\subsection{La traduction de L'étranger par Ilana Habermann : l'écriture blanche au premier degré}

En 1985, Ilana Habermann publia sa traduction de L'étranger ${ }^{9}$ qui vint remplacer I'ancienne traduction effectuée par le poète et traducteur Aaron Amir. Le parti pris méthodologique adopté par la nouvelle traductrice consiste à transposer la voix blanche de Meursault en un hébreu tout ce qu'il y a de plus prosaïque et quotidien qui contraste fortement avec l'option d'Aaron Amir, soucieux de ne pas infecter son style de vulgarisme, sauf dans les répliques véristes. Point de cet écart frappant qui caractérisait le style du poète Ratosh traduisant la chronique de La peste en un hébreu constellé d'élégances bibliques. II n'est du reste pas fortuit que les traductions élégantes et même guindées de La peste par Yonathan Ratosh et de L'étranger première version par Aaron Amir soient l'œuvre de poètes. II est difficile de renoncer de gaîté de cœur aux charmes incantatoires de la langue hébraïque pour en faire un reflet du style délibérément neutre ou trivial de Camus romancier.

Sous la plume d'Ilana Habermann, la langue oscille entre la neutralité stylistique et la trivialité dès lors que Camus fait parler ses personnages. II n'y a donc point de cette homogénéisation stylistique que nous avons vue à l'œuvre dans le cas de la traduction de La peste.

Cette transposition fidèle des registres stylistiques du texte-source dans la langue-cible n'est pourtant pas asservie par trop de littéralisme. Loin de transposer mot à mot le texte de Camus, Ilana Habermann s'efforce de trouver pour chaque tour idiomatique du français un équivalent dynamique. Autant dire que la démarche communicative l'emporte sur l'option informative et sémantique. M ais comme les temps ont changé, le style guindé ne peut plus passer pour l'analogon de la voix blanche, de sorte que le français dépouillé devient de l'hébreu dépouillé. L'écriture blanche est donc rendue par une autre écriture blanche, au premier degré.

Pour illustrer notre propos, nous voudrions citer quelques exemples de cet art de transposer le français simple en un hébreu non moins simple qui n'en est pourtant pas la mise à plat verbum pro verbo. À la vérité, le secret de l'élégance à laquelle llana Habermann est parvenue dans sa traduction tient à son talent de tranposer les phrases plutôt que les mots qui la composent. La phrase de l'original français est analysée dans son contexte stylistique et pragmatique et, une fois identifiée comme liée à telle ou telle situation communicative, l'équivalent dynamique en hébreu parlé s'impose de lui-même.

C'est ainsi que la phrase triviale de Céleste, «on n'a qu'une mère» $(I, 1)$, est rendue par le non moins trivial אמא יש רק אחת. Pour bien faire comprendre l'effet stylistique produit par cette trouvaille, signalons que le premier traducteur, Aaron Amir, avait transposé cette même phrase par אין כמו אם. Bien qu'elle soit d'une élé- 
gance laconique, cette transposition manque un peu de vie et reflète imparfaitement le registre stylistique dans lequel Camus a voulu faire parler Céleste.

Un autre exemple de cette dextérité à trouver l'équivalent global du français dans le même registre stylistique et avec la même intention pragmatique nous est fourni par la traduction de la phrase «Pendant tout ce temps, le concierge a parlé» (ibid.). Ilana Habermann rend cette courte proposition par במשך כל הזמן הזה דיבר השוער במדר. C'est sous l'influence du complément circonstanciel de temps «pendant tout ce temps» que la traductrice n'a pas hésité à surtraduire le verbe «il a parlé» par . De cette façon, elle a compensé la pauvreté du système temporel du verbe hébreu par le procédé stylistique de la répétition du verbe. Signalons d'ailleurs que cette expression, דיבר ודיבר, est très vraisemblablement le calque d'un tour syntaxique du yiddish qui consiste à répéter le verbe à la même forme ou du moins le participe passé pour marquer le caractère itératif ou intensif de l'action. On pourrait

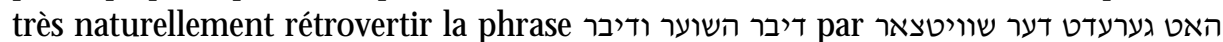
Bien entendu, cette influence du substrat yiddish s'est exercé sur le plan de la langue plutôt qu'au niveau de la parole individuelle. Mais le fait ne mérite pas moins d'être relevé.

L'hébreu classique pur de toute influence du vernaculaire yiddish, I'hébreu d'un Ratosh somme toute, aurait employé ici un tour consistant à répéter la même racine verbale, mais avec un statut morphologique différent (verbe conjugué + infinitif absolu : דבר דיבר). La répétition du verbe à la forme conjuguée témoigne de l'ancrage de la langue d'Ilana Habermann dans I'hébreu contemporain, un hébreu truffé d'influences syntaxiques des langues européennes, qu'elles soient juives comme le yiddish ou gentilles, comme le russe, l'allemand ou l'anglais.

Le recours à un idiomatisme yiddish transposé en hébreu moderne permet d'exprimer analogiquement le caractère absolu de «il a parlé» qui désignel'action considérée pour elle-même, sans aucune délimitation temporelle ou aspectuelle ni même de complément qui la précise.

Dans les deux exemples qui précèdent nous avions affaire à une réplique au style direct dans le cas de la phrase sentencieuse de Céleste, et au monologue intérieur de Meursault, dans le cas du verbe «il a parlé». II faudrait mentionner un exemple tiré d'un passage au style indirect libre. Dans le même chapitre $(I, 1)$, on trouve la phrase «M ais naturellement, ce n'était pas la même chose». Cette attaque adverbiale encore accentuée par l'emploi de la conjonction «mais» est fort astucieusement rendue par une exclamation passe-partout de l'hébreu parlé:

מה פתאום, זה לא אותו דבר.

Non seulement l'exclamation מה פתאום est un équivalent pragmatique judicieux de «mais naturellement», mais en plus il y a même un effet d'imitation sonore du «mais» au moyen du pronom interrogatif מה?. L'écart par rapport à la formulation du texte original et le passage du style indirect libre au style direct sont compensés par un effet de mimétisme des sonorités du texte original.

On pourrait citer d'autres trouvailles stylistiques du même genre qui confèrent nature et fraîcheur à la traduction d'Ilana Habermann. Ainsi, Iorsqu'Emmanuel propose à Meursault de grimper sur un camion en marche, il lui lance la phrase «Si on y allait?». Et Ilana Habermann de traduire judicieusement cette invitation à 
l'emporte-pièce par un très véridique עולים? qui a en outre l'avantage de répercuter quelque peu la structure syllabique de la forme verbale allait.

D'une manière générale, Ilana Habermann est très sensible aux sonorités du texte. Nous avons vu ci-dessus qu'à deux reprises son hébreu reproduit dans la mesure du possible le matériau phonétique du français. II s'agit alors d'une allitération translinguistique qui resserre le lien entre texte-source et texte-cible. Mais il arrive aussi qu'au sein même du texte-cible, Ilana Habermann prenne l'initiative d'inventer des allitérations qui ne correspondent pas à des allitérations du textesource. Citons par exemple le cas du syntagme nominal «cette terre rousse et verte» traduit par האדמה האדומה והירוקה הזאת (l,1). Ici la rime interne entre le substantif est en outre une figure étymologique. Comment justifier cette débauche de raffinements stylistiques et la concilier avec ce que nous constations tout à l'heure à propos de la démarche communicative d'Ilana Habermann, soucieuse de transposer l'esprit du texte et sa sobriété ascétique? En fait, on peut considérer que cette audace stylistique de la traductrice est motivée par le désir de compenser un insuffisance ponctuelle de l'hébreu par un effet de surtraduction. En effet, l'hébreu ne possède pas le moyen qu'a le français de distinguer entre la couleur «rouge» et la couleur «rousse». L'adjectif אדמוני ne saurait convenir, car il est spécialisé dans la désignation des individus aux cheveux roux. La transposition métaphorique à la terre risquerait d'être incomprise par un lecteur israélien et ferait l'effet d'une licence poétique. Cette insuffisance due à une différence dans le découpage des champs sémantiques d'une langue à l'autre est néanmoins compensée par le jeu rythmique et étymologique entre אדמה et אדומה.

Parfois l'emploi d'une allitération ou d'une rime interne ne s'explique pas par un désir de compenser quelque chose de précis. C'est le cas dans la phrase qui suit le passage que nous venons de citer. Le syntagme «comme une trêve mélancolique» y est traduit par כמין הפוגה נוגה, où l'on trouve un effet de rime interne entre הפוגה et נוגה. Le recours à ces raffinements stylistiques ne vient pas briser la sobriété élégante recherchée par Ilana Habermann. L'allitération et la rime interne constituent le charme inhérent à la langue hébraïque, même dans ses registres les plus humbles, de la même façon que la langue française tire sa beauté des modulations et des assonances que lui permet son très riche diapason de voyelles. Tout se passe comme si Ilana Habermann avait tenu à transposer la beauté de la langue française (essentiellement vocalique) par celle de la langue hébraïque (fondée sur les effets rythmiques de la rime interne, de l'allitération et sur les jeux sémantiques de la figure étymologique).

Au-delà de ces consi dérations générales qui suffiraient en elles-mêmes à justifier le recours au procédé de la compensation, il faut noter que l'expression «comme une trêve mélancolique» n'est pas anodine dans le roman : elle réapparaît au tout dernier paragraphe $(I I, 5)$, comme un écho du premier chapitre $(I, 1)$. C'est parce qu'elle a senti que cette comparaison constituait un jalon important de la structure dramatique de L'étranger que la traductrice a pris le parti d'en rehausser la traduction par un effet de style et de faire jouer la rime interne entre le substantif et l'adjectif, comme pour mieux souligner de façon emblématique qu'en elle-même cette expression répétée au dernier chapitre fonctionne comme une sorte de rime permettant de mettre en communication le prologue et l'épilogue. 


\subsection{La traduction d'Aviva Barak : une prose solennelle pour un poème en prose}

En traduisant Noces et L'été, Aviva Barak ne se heurtait pas au défi consistant à transposer la voix blanche du romancier Camus en hébreu. Le style de l'écrivain est ici marqué par un lyrisme chaleureux et par une propension sensuelle à la description qui fait totalement défaut dans La peste, où la ville d'O ran nous est présentée symboliquement comme une ville «sans fleurs», ou dans L'étranger, roman de l'absence et du détachement.

Nous avons vu ci-dessus que Ratosh avait paradoxalement rendu la prose dépouillée de Camus romancier par un style apparemment emphatique. En réaction contre ce décalage justifié ou non, Ilana Habermann avait pris le parti de traduire l'écriture blanche par une écriture hébraïque tout aussi blanche. Cette recette stylistique qui lui avait si bien réussi dans le cas de L'étranger fut moins heureuse lorsqu'dle s'attaqua à la prose lyrique de Camus, poète méditerranéen. On se trouve alors confronté au même genre de divergence stylistique que ci-dessus, mais en sens inverse: au lieu de traduire la voix blanche par un style élégant, comme le faisait Ratosh, Ilana Habermann a traduit «Retour à Tipasa», exemple du style lyrique de Camus, par un style assez neutre qui n'est pas toujours très adéquat.

Le hasard des rééditions a voulu que ce même essai soit retraduit quelques années plus tard dans le cadre de la traduction de L'étépar Aviva Barak ${ }^{10}$. La comparaison entre les deux versions, l'une prosaïque et «blanche», I'autre plus poétique et recherchée, permettra de comprendre que les recettes qui convenaient pour traduire le monologue de Meursault ne peuvent plus fonctionner dès lors qu'il s'agit de transposer la voix de Camus lui-même.

Ajoutons en outre que la démarche communicative se justifiait parfaitement dès lors qu'il saaissait de traduire les situations assez simples de L'étranger. Mais dans «Retour à Tipasa», il n'y a plus de contexte pragmatique ou socioculturel susceptible de guider le traducteur dans le choix de l'équivalent le plus approprié. Force est donc de traduire au plus près et d'adopter une démarche informative et sémantique. Ajoutons à cela que la prose des essais lyriques de Camus a le statut d'un verbe poétique. II perd donc de sa transparence et de sa neutralité et il importe d'en transposer la dimension phatique.

De la même façon, la traduction de L'homme révolté par Zvi Arad est moins bien réussie que sa traduction de La chute. Car tout comme dans L'étranger, le style de La chute se caractérise par son recours délibéré à des clichés et à des formules toutes faites. Bien entendu, il ne s'agit pas du même registre stylistique puisque La chute est écrite en une parodie de la rhétorique enlevée des plaidoyers, avec les effets de manche qui la caractérisent. Mais mutatis mutandis, le traducteur avait beau jeu de trouver pour chaque phrase et chaque tournure un équivalent dynamique. En revanche, le raisonnement philosophique de L'homme révolté tout comme l'emphase lyrique de Noces et L'été ont tous deux quelque chose d'erratique qui se laisse difficilement transposer par des équivalents préexistants en hébreu. Le traducteur en est donc réduit à adopter une démarche informative et à se concentrer sur chaque mot en particulier, ce qui rend la traduction assez laborieuse et heurtée.

II semble, que sur ce chemin de la littéralité, Ilana Habermann ait été moins à I'aise qu'Aviva Barak. Cette dernière a en effet réussi à concilier la fidélité au texte- 
source, dont elle a transposé autant que possible les connotations expressives, avec le souci d'écrire dans un bel hébreu. Citons par exemple les deux traductions successives de la phrase «Le soir, dans les cafés violemment éclairés où je me réfugiais, je lisais mon âge sur des visages que je reconnaissais sans pouvoir les nommer ».

Ilana H abermann :

בערב, בבתי הקפה המוארים באור עז שמצאתי לי בהם מקלט, קראתי את גילי על פנים ולא ידעתי את שמבתי הקפיה במותי

Aviva Barak:

לעת ערב, מצאתי לי מחסה בבתי קפה מוצפים אורות עזים, ואת גילי קראתי על פני אנשים שהכרתי לפנים ועתה לא ידעתי את שמם.

Des deux traductions, celle d'Aviva Barak apparaît comme la plus recherchée. לעת ערב Outre de menus raffinements lexicaux, comme le complément de temps au lieu du prosaïque בערב, il faudrait mentionner que la proposition subordonnée relative «où je me réfugiais» est transposée par la proposition indépendante מצאתי לי מחחסה. Ce truc de traducteur évite de maintenir une enclave syntaxique qui risquerait d'être fort disgracieuse du fait de la saturation des déterminants de «cafés»: «cafés violemment éclairés» (premier déterminant) «où je me réfugiais» (deuxième déterminant). Ilana H abermann a traduit l'un après l'autre les deux déterminants de «cafés» de sorte que le déterminant המוארים באור עז est suivi immédiatement de la relative assez gauche שמצאתי לי בהם מקללט. Cette accumulation de déterminants, qui passe bien en français puisque l'adverbe «violemment » est antéposé au participe «éclairé», devient lourde en hébreu où l'on est obligé de traduire l'adverbe par un syntagme nominal (באור עז) nécessairement postposé. Pour éviter cette cascade de déterminants postposés aux substantifs בתי קפה, Aviva Barak a donc eu la bonne idée de transformer la relative française en une proposition indépendante, ce qui lui a permis de rééquilibrer agréablement la répartition des termes dans la phrase.

La raison de cette supériorité relative de la traduction de «Retour à Tipasa »par Aviva Barak sur celle d'Ilana Habermann tient en partie au fait qu'il est en un sens moins difficile de reprendre une traduction déjà effectuée, quitte à en prendre le contrepied, que de se mesurer pour la première fois à un texte jamais traduit auparavant. Cet avantage apparaît a contrario à travers la traduction des autres essais contenus dans $\mathrm{N}$ oces et dans L'été qui ne s'est pas appuyée sur la leçon d'un passé récent et qui s'est donc trouvée aux prises avec le vide vertigineux de la page blanche. Ilana Habermann, qui avait su tirer la leçon des défaillances de la traduction de L'étranger par Aaron Amir pour produire sa propre traduction, assurément beaucoup plus lisible, a enduré à son tour le sort qu'elle avait fait subir au premier traducteur de L'étranger: sa traduction de «Retour à Tipasa» a servi de tremplin à la traduction mieux léchée d'Aviva Barak.

Signalons enfin que dans un tout autre registre, Aviva Baraq s'est départi de l'option informative en transposant de la façon la plus communicative qui soit les dialogues qui agrémentent de temps à autre N oces et L'été. À titre d'illustration, nous voudrions citer un extrait de L'été à Alger qui constitue l'un des chapitres de N oces. II s'agit d'une reproduction vériste du parler pataouète de Bab-el-Oued. Voici tout d'abord la citation du texte d'Albert Camus: 
Alors Coco y s'avance et y lui dit: «Arrête un peu, arrête. » L'autre y dit: «Q u'est-ce qu'y a?» Alors Coco y lui dit: «Je vas te donner des coups. - À moi tu vas donner des coups?»Alors y met la main derrière, mais c'était scousa. Alors Coco y lui dit: Mets pas la main darrière, parce qu'après j'te choppe le 6-35 et t'y mangeras des coups quand même.»

L'autreil a pas mis la main. Et Coco, rien qu'un, y lui a donné- pas deux, un. L'autre il était par terre. «Oua, oua», qu'y faisait. Alors le monde il est venu. La bagarre, elle a commencé. $Y$ en a un qui s'est avancé à Coco, deux, trois. Moi j'y ai dit: "Dis, tu vas toucher à mon frère? - Qui, ton frère? - Si c'est pas mon frère, c'est comme mon frère. » Alors j'y ai donné un taquet. Coco y tapait, moi je tapais, Lucien y tapait. Moi j'en avai un dans un coin et avec la tête: «Bom, bom. » Alors les agents y sont venus. Y nous ont mis les chaînes, dis. La honte à la figure, j'avais, de traverser tout Bab-el-Oued. Devant le Gentleman's bar, y avait des copains et des petites, dis. La honte à la figure. Mais après, le père à Lucien y nous a dit: «Vous avez raison. »

C'est avec beaucoup de brio et avec une intuition très sûre qu'Aviva Baraq a transposé ce spécimen du parler pataouète dans le langage des classes défavorisées de la société israélienne, et plus précisément dans le sociolecte des jeunes Israéliens d'origine marocaine. Voyez plutôt:

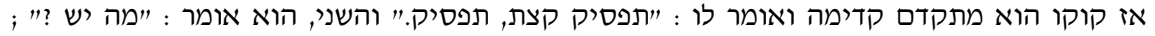

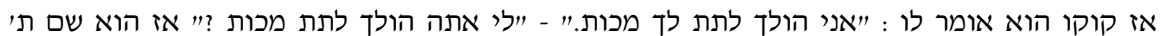

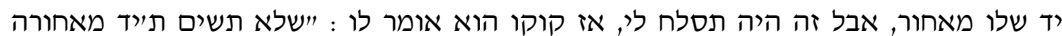

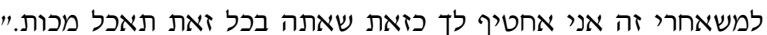

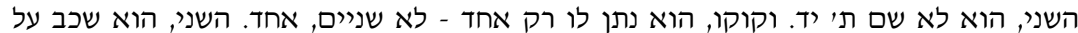

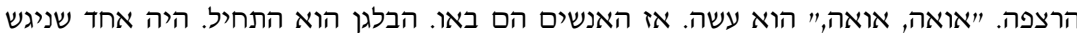

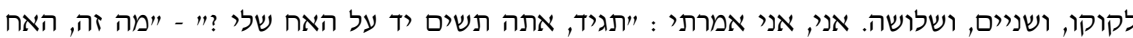

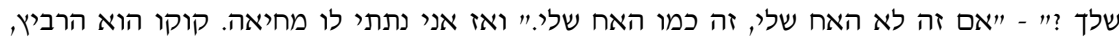

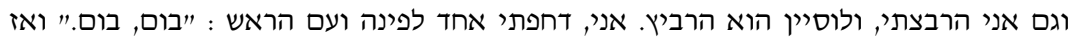

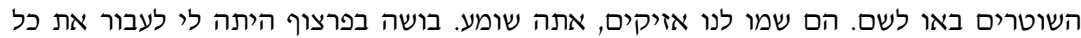

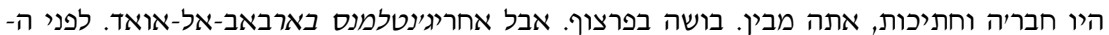
זה, האבא של לוסיין אמר לנו : "אתם אתרו. בות אלה שצודקים".11
}

C'est sur cette note ensoleillée que je terminerai cette confrontation des diffé rents styles de traduction appartenant à deux générations différentes de la République des Lettres néo-hébraïques et représentant tantôt une approche communicative et dynamique de la traduction, tantôt un méthode informative et sémantique.

$M$ ais ces termes de traductologie risquent peut-être de choquer les oreilles allergiques au jargon des linguistes. On peut donc les remplacer avantageusement par une terminologie plus littéraire, celle de Goethe. Dans une note apposée à sa traduction de quelques poèmes persan $s^{12}$, le grand poète allemand distingue un premier type de traduction qui est une mise à plat littérale du texte, un deuxième type qui recourt aux «belles infidèles» et enfin un troisième type qui est «l'ultime et le meilleur » «der letzte und der beßte») puisqu'il consiste pour le traducteur bien doué à acclimater dans la langue-cible certaines particularités de la langue-source. Si l'on essaie d'appliquer cette tripartition au cas des traductions de Camus en hébreu, il apparaît que les traductions de La peste et de L'étranger ressortissent au deuxième type. En revanche, les essais contenus dans $N$ oces et dans L'été sont transposés selon les principes de la mise à plat, même si Aviva Barak parvient souvent à concilier la fidélité au textesource avec le souci de produire un texte-cible non dépourvu de recherche stylistique. Quant aux traductions de L'envers et l'endroit ${ }^{13}$ et de Lettres à un ami allemand ${ }^{14}$ par 
Adina Kaplan, elles sont d'un littéralisme si extrême qu'elles en deviennent parfois inexactes. Ainsi, à la fin de l'introduction à l'édition italienne des Lettres à un ami allemand, Adina Kaplan traduit «vous autres Allemands», «vous autres nazis», «nous autres Français» et «nous autres, Européens libres»par אתם הגרמנים האחרים, אנחנו הצרפתים האחרים ,אתם הנאצים האחרים et אנחנו האחרים, האירופאים החופשיים. Ce faisant, elle est tombée dans un contresens, car dans les expressions «nous autres» et «vous autres», I'adjectif apposé «autres» a perdu sa valeur sémantique pour devenir un simple mot-outil. Certes le français n'a pas poussé cette tendance aussi loin que le castillan ou l'occitan où nosotros, vosotros et nautre, vautre sont devenus la seule et unique manière d'exprimer le pronom sujet de la quatrième et de la cinquième personnes. Mais quand bien même nous autres et vous autres ne seraient qu'une variante stylistique de nous et vous, la variation porte sur l'emphase expressive et non sur l'information sémantique. Un peu plus de distance par rapport à la formulation du texte original aurait peut-être permis d'éviter cette erreur.

II ne reste plus qu'à souhaiter l'émergence du troisième type de traductions, «l'ultime et le meilleur», celui qui parviendra à concilier la fidélité au texte original avec l'audace créatrice dans la langue d'arrivée, de sorte que le traducteur se fasse véritablement l'émule de l'auteur du texte qu'il convertit dans sa propre langue. Mais cette prospective n'est peut-être qu'une hypothèse de travail ou un rêve de poète. En attendant, il importe de revenir à des réalités plus concrètes et d'analyser la façon dont le contexte culturel dans lequel baignent les romans de Camus a été transposé à l'intention d'un autre public dans un autre pays.

\section{LA CONVERSION DES REALIAS ET DES RÉFÉRENCES CULTURELLES}

Bien que Camus ne soit pas un auteur entiché de couleur locale, il reste que même dans sa prose la plus neutre on discerne l'empreinte d'une certaine époque et d'un certain contexte culturel. Afin d'apprécier à leurs justes mesures les efforts accomplis par les traducteurs israéliens pour rendre compréhensible ce qui est irréductible à l'universalité de l'expérience humaine, nous comparerons occasionnellement des traductions de Camus en arabe, en castillan, en allemand en anglais.

\subsection{Jean-Baptiste Clamence peut-il parler autre chose que le français?}

Le bavard impénitent qui monologue dans La chute est une caricature bien enlevée de l'esprit parisien, au point qu'on a peine à croire que ses circonvolutions oratoires et ses simagrées cabotines puissent être exprimées dans une autre langue que celle de Voltaire. Et pourtant deux traducteurs de La chute ont réussi le tour de force de transposer la longue péroraison de Clamence dans des langues fort éloignées du français: Zvi Arad en hébreu ${ }^{15}$ et Anis Zakis Hasan en arabe ${ }^{16}$. La comparaison entre ces deux versions, I'une hébraïque et l'autre arabe, présente un certain intérêt, car elle permet de mesurer ce qui réunit ou ce qui sépare le traducteur israélien du traducteur arabe dans la conversion du français (peut-être à travers le truchement de l'anglais, comme certains indices tendent à le suggérer) en une langue sémitique. Aussi bien est-il à première vue plus légitime de comparer une traduction hébraïque avec une traduction arabe, étant donné la parenté génétique entre les deux langues et la 
différence qui sépare leur structure grammaticale des langues indo-européennes.

Dans ces conditions, la traduction d'une phrase comme «Ah! je vois que vous bronchez sur cet imparfait du subjonctif » s'avère très problématique, d'autant plus qu'il sagit d'une reférence métalinguistique explicite à un fait de langue inconnu de l'hébreu et de l'arabe. Confrontés à ce problème, les deux traducteurs ont réagi différemment: Zvi Arad a traduit selon l'esprit, tandis que son homologue libanais a respecté la lettre du texte, quitte à procéder à une légère transposition. Le traducteur israélien a interprété le sens du terme «imparfait du subjonctif» dans le contexte et il a transposé la connotation de ce mot plutôt que sa dénotation précise en traduisant «imparfait du subjonctif» par les périphrases מכמני הלשון שלי, puis דקדוקי עניות שלי בלשון.

De son côté, Anis Zaki Hasan a escamoté la connotation de ce mot en tant qu'il fait référence à la pédanterie scrupuleuse et au purisme tâtillon et il l'a transposé par une autre catégorie grammaticale qui n'est plus un mode, mais la conjonction de subordination idhâ. Pour cela, il a dû ajouter à la proposition «sans qu'aussitôt j'en fisse ma société», à propos de laquelle Jean-Baptiste Clamence critique son propre langage, une tournure qui fait apparaître la conjonction idhâ: fa-idhâ kân dhalika hamâqah.... «et si cela était une sottise... », proposition subordonnée conditionnelle laissée en suspens. Mais ce n'est pas lui qui a eu l'initiative de cette interpolation: dans la traduction anglaise de Justin O'Brien (Camus 1957a), on trouve un ajout identique: «if that be foolish... » Et comme la traduction anglaise est de quatorze ans antérieure à la traduction arabe, force est d'admettre que le traducteur libanais a pris exemple sur son homologue anglo-saxon et non l'inverse. Dans la stratégie de traduction de Justin O'Brien, cet ajout se justifie par la valeur du subjonctif anglais, survivance morphologique qui n'apparaît guère que dans les propositions conditionnelles. Pour faire apparaître un subjonctif, il fallait lui donner un support adéquat et c'est cette proposition suspendue en l'air par une anacoluthe qui a fait l'affaire. Anis Zaki Hasan, confronté au même problème que ses prédécesseurs a retenu la recette de Justin O'Brien et il a traduit assez littéralement «if that be foolish» par «fa-idhâ kan dhalika hamâqah » «et si c'était une sottise... » Et comme l'arabe n'emploie pas le subjonctif dans ce genre de proposition conditionnelle, il a déplacé son attention du mode verbal à la conjonction de subordination idhâ.

Ainsi donc le traducteur israélien et son homologue libanais ont réagi différemment devant une incompatibilité analogue: Zvi Arad a préféré contourner l'obstacle en élargissant l'acception du signifié, tandis qu'Anis Zaki Hasan a procédé à une équation entre un mode français (ou anglais, si tant est quel'anglais ait interféré dans le passage du français à l'arabe) et une conjonction arabe, sans se préoccuper de l'intention connotative et expressive qui a poussé Camus à cette digression métalinguistique.

Signalons du reste que le procédé par lequel Zvi Arad a diluéle signifié du terme «imparfait du subjonctif » en le traduisant par une formule faisant référence au beau style (מכמוני הלשון שלי /דקדוקי עניות שלי בלשון) a été utilisé par les traducteurs allemand (Camus 1957b) et mexicain (Camus 1956). Pourtant l'allemand possède le Konjunktiv II, équivalent fonctionnel du subjonctif imparfait, cependant que le castillan dispose de deux imparfaits du subjonctif dont l'un - la forme en -se - jouit d'un statut stylistique exactement équivalent à l'imparfait du subjonctif français ${ }^{17}$. $M$ ais il faut dire qu'en allemand au moins, I'emploi du Konjunktiv II est certainement 
plus naturel et moins châtié que le subjonctif imparfait français. Pour préserver la connotation que Camus a conférée au terme «imparfait du subjonctif », le traducteur allemand a donc recouru à l'expression générique umständliche Formulierung, en traduisant la proposition «sans qu'aussitôt j'en fisse ma société» par une tournure syntaxique dont le caractère châtié tient autant sinon plus au lexique qu'à l'emploi du Konjunktiv II : ohne daß ich sogleich vertrauten Umgang mit ihm gepflogen hätte.

Quant au traducteur mexicain, il ne sest pas donné beaucoup de peine: il a traduit la première occurrence du terme par «el hablar elegante» et il en a purement et simplement escamoté la seconde occurrence, de sorte que la connotation que Camus lui a prêtée s'évanouit complètement. Cela est d'autant plus frappant que pour traduire la phrase «Quand je vivais en France, je ne pouvais rencontrer un homme d'esprit sans qu'aussitôt j'en fisse ma société», il a employé un subjonctif en -se: «Cuando vivía yo en Francia, me ligaba inmediatamente con todo hombre de ingenio que encontrase». Mais ne voulant s'appesantir sur des considérations métalinguistiques, il a dilué le signifié du terme grammatical en une formule passepartout.

À la lumière de cet exemple se vérifie un paradoxe de la traduction: il arrive parfois que le processus de conversion d'une langue à l'autre soit d'autant plus difficile que la langue-cible est génétiquement proche de la langue-source. En un sens, traduire Camus en allemand, en anglais ou à plus forte raison en castillan pose parfois plus de problèmes que d'en donner une version hébraïque ou arabe, car le processus de transposition est entravé par une multitude de faux amis: le traducteur ănglo-saxon n'a-t-il pas été contraint de créer une phrase de toutes pièces pour loger son subjonctif be?

Ainsi donc Zvi Arad se comporte ici comme les traducteurs allemand et mexicain puisqu'il transpose la connotation et escamote de la dénotation précise au lieu de sacrifier la connotation à la dénotation, comme le fait le traducteur libanais. Ce rejet du littéralisme est la marque de l'ancrage de ce traducteur israélien dans une tradition de traduction tout ce qu'il y a de plus occidentale ${ }^{18}$, à l'opposé du littéralisme qui caractérise la praxis de la traduction en Orient. En l'occurrence, ce littéralisme nous a fourni la pièce à conviction qui tend à prouver qu'Anis Zaki Hasan est passé par le truchement partiel ou total du texte anglais, puisqu'il a reproduit mot pour mot l'interpolation de Justin O'Brien.

Du point de vue lexical, on remarquera une intéressante rencontre entre le traducteur israélien et son homologue libanais. Elle concerne la traduction du mot «genièvre». Tous deux l'ont traduit par le mot anglais gin : ג en hébreu, jin en arabe. Cette apparente liberté de traduction est en fait fondée sur l'étymologie du mot anglais gin qui n'est autre que l'abréviation d'un plus ancien, geneva (prononcez /ginívว/), lequel est un emprunt au néerlandais jenever / genever ${ }^{19}$. M ême si le gin anglais n'est pas parfumé avec des baies de genièvre comme le genièvre hollandais, l'idée de traduire le mot «genièvre» par son avatar «gin » permet d'éviter le recours à une note de traducteur. Du reste, il n'est pas exclu que cette trouvaille soit imputable une fois de plus au truchement de la traduction de Justin O'Brien où «genièvre» est traduit comme de juste par «gin ».

Pour achever cette comparaison entre la traduction de La chute en hébreu et en arabe, mentionnons que le traducteur libanais Anis Zaki Hasan, au demeurant si littéral, s'est départi de sa fidélité à l'égard du signifié chaque fois que le texte de Camus 
fait référence au judaïsme, qu'il soit biblique ou contemporain. Le passage où JeanBaptiste évoque les «soixante-quinze mille juifs déportés ou assassinés » est purement et simplement censuré. Les «enfants de Judée» deviennent des atfâl «enfants» et Rachel se voit désignée de façon anonyme par le terme générique par al-marâh «la femme». Quant au mot «Sadducéen », il est périphrasé par kâfir bi-I-qayimah «reniant la résurrection des morts». Pour éviter de faire explicitement référence à une réalité historique juive, le traducteur libanais a retenu de la signification du mot «Sadducéen » un sème qui n'est pas pertinent dans le contexte. Si Camus a évoqué ce courant du judaïsme ancien, ce n'est pas pour se référer au dogme de la vie future, mais bien plutôt par rapport au statut de cette caste sacerdotale dans la société juive de l'époque du Second Temple.

Ainsi donc la voix de Jean-Baptiste Clamence a été transposée à peu près sans encombre en hébreu et en arabe. $M$ ais dans cette dernière langue, le juge-pénitent des bars d'Amsterdam a été censuré outrageusement par un traducteur tendancieux. On mesure à travers cet exemple l'impact que le contexte politique et culturel peut exercer sur l'acte de traduction qui est non seulement un passage de langue à langue, mais aussi une façon de faire communiquer des cultures différentes.

\subsection{De la nécessité de transposer fidèlement la dimension contingente du raisonnement philosophique}

Nous continuerons ce passage en revue des problèmes liés à la transposition d'une culture à une autre par l'examen de certaines maladresses commises par des traducteurs peu attentifs à l'ancrage concret du texte de Camus. M ais il est bien entendu que ces petits défauts sont purement involontaires et qu'ils ne doivent pas être mis sur le même plan que la mesquine censure mise en œuvre par le traducteur libanais.

Prenons le cas du M ythe de Sisyphe par Zvi Arad²0. À lui seul le titre המיתוס של סיזיפוס se signale comme une transposition très plate du titre français. Puisque I'hé breu possède un très large éventail de prépositions, il arrive souvent qu'à la préposition multivalente de du français correspondent toutes sortes d'autres prépositions en hébreu. En choisissant de transposer de par של, Zvi Arad a commis une inexactitude, car on pourrait croire qu'il a voulu exprimer un rapport de possession entre le mythe

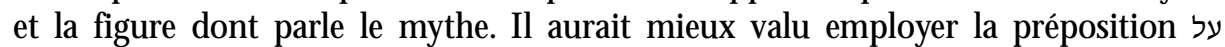
ou bien la préposition במיתוס על סיסיפוס) ou (מעשה בסיסיפוס). En outre l'orthographe מיזיפוט n'est pas adéquate, car elle reflète la prononciation francisée du nom grec $\Sigma$ ıovфos. M ême si le texte-source est un essai philosophique français, rien ne justifie cette médiation du français dès lors qu'il sagit de transposer un nom propre grec.

Plus grave encore est le faux sens qui apparaît à la page 25 de cette traduction hébraïque du M ythe de Sisyphe: le mot «fourmilier » qui désigne un curieux animal d'Amérique du Sud est traduit par קן נמלים, «fourmillière», au terme d'une lecture inattentive du texte-source ou peut-être à cause d'une connaissance insuffisante de la langue-source. Signalons du reste que dans la traduction américaine du Mythe de Sisyphe, le mot «fourmilier » est rendu tout aussi malencontreusement par «anthill» (Camus 1955: 13). Simple rencontre provoquée par une difficulté du français qui utilise deux mots assez semblables pour désigner le nid de fourmis et l'écumeur de fourmis? Ou bien indice d'une interférence de l'anglais entre le texte-source français et le texte-cible hébreu ? Quoi qu'il en soit, ces deux traductions du M ythe de Sisyphe 
sont tombées dans le piège qui consiste à ne pas prêter attention à la matérialité concrète et à l'ancrage contingent du verbe camusien. Ces essais philosophiques ont beau débattre de sujets abstraits et généraux, ce n'est pas une raison pour se désintéresser de l'épaisseur du signifiant. Puisque Camus soignait amoureusement la forme de ses écrits, c'est une double trahison que de le traduire de façon si désinvolte: trahison par rapport à son message et trahison par rapport à l'estime dans laquelle Camus tenait le verbe artistique.

\subsection{Des mots dont le référent s'est estompé}

Toute écriture romanesque implique une certaine représentation de la réalité qui englobe parfois les objets les plus contingents. M ême des romans aussi ascétiques que La peste et L'étranger n'échappent pas à la règle. Or l'O ran de La peste ou l'Alger de L'étranger constituent un monde doublement révolu: d'abord parce que l'indépendance de l'Algérie a court-circuité la synthèse culturelle qui était en train de mûrir sur ces rivages et ensuite parce qu'entre les années 1940 et 1950 et la fin des années 1990, le monde où nous évoluons quotidiennement a subi des modifications de toutes sortes, à commencer par des aspects aussi immédiats que la mode ou les techniques.

Le décalage qui s'accuse au sein même de la langue francaise entre le signifié et le reférent, voire même entre le signifiant et le signifié (il est des signifiants qui ne disent plus rien aux lecteurs) est encore plus accentué lorsqu'il sagit de traduire les realias de l'Algérie française d'avant l'indépendance dans la langue rénovée d'un jeune État dont la mémoire collective est très fragmentée par la diversité d'origines des habitants, les références communes ne constituant en somme qu'un minimum élagué réduit à sa plus simple expression.

Prenons par exemple le cas des mots «canotier » (L'étranger I,6) et «panama» (ibid. II,3). Ces couvre-chefs estivaux ne sont guère plus que des mots dans la conscience linguistique des jeunes Français d'aujourd'hui. À plus forte raison le sont-ils dans une société américani sée où les seuls chapeaux connus sont la casquette de base-balleur, le béret du soldat ou à la rigueur le borsalino des ultra-orthodoxes. Pour traduire ces termes techniques de la modisterie, Ilana Habermann a pris le parti de recourir à une périphrase descriptive: elle a donc rendu «canotier» par בובע קש et "panama»" ce dernier équivalent ayant été employé pour «canotier» par Aaron Amir dans sa traduction de La mort heureuse $(I I, 4)$. Plus près de la France, les traducteurs allemands n'ont pas fait mieux. Dans la version tudesque de L'étranger (Camus 1981), le «canotier» devient tout simplement un Strohhut, un «chapeau de paille» sans autre précision, tandis que «panama» est maintenu tel quel. De la même façon, le traducteur américain ${ }^{30}$ avait rendu «canotier » par «straw hat » et gardé le terme «panama » (Camus 1946).

Cet estompement de l'ancrage historique et culturel des romans de Camus n'a rien qui doive nous gêner, car les traducteurs ont délibérément choisi de recourir à ces périphrases. Bien loin de trahir le texte, ils en préservent l'éternelle jeunesse en transformant la variable éphémère pour mieux en laisser subsister l'essence anhistorique.

Citons un autre exemple visant à pallier l'opacité d'un signifiant trop ancré dans une réalité étrangère. II s'agit du terme «prie-Dieu » qui apparait dans La peste $(I I, 3)$ : 
«quelques auditeurs, après une seconde d'hésitation, se laissèrent glisser de leur chaise sur leur prie-Dieu ». Yonathan Ratosh a contourné élégamment l'obstacle en traduisant cette phrase par

כמה מקהל המתפללים החליקו מעל מושבם, לאחר שניה של היסוס, אל האנלוגין.

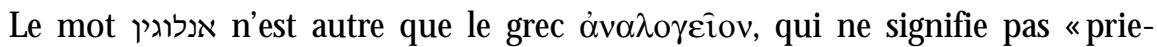
Dieu», mais «pupitre». C'est au prix d'une légère inexactitude peut-être volontaire queYonathan Ratosh a réussi à transposer ce terme technique qui relève d'une réalité inconnue du public israélien. À titre de comparaison, signalons que dans sa traduction de La peste, Stuart Gilbert a purement et simplement escamoté le problème posé par la traduction de «prie-Dieu » et il a traduit la phrase de façon communicative et dynamique par «Some of the worshippers slipped forwards from their seats on to their knees» (Camus 1948).

\subsection{Comment transposer la couleur locale algérienne?}

Les simplifications, les périphrases ou les substitutions décrites ci-dessus à propos des accessoires d'une mode obsolète sont inévitables et elles n'ont en somme guère d'incidence tant qu'il n'est question que de chapeaux ou de prie-Dieu. L'amalgame devient un peu plus gênant lorsque l'incapacité à transposer certaines realias reflète un blocage culturel. Je veux parler de la transposition des termes arabes qui affleurent dans l'œuvre de Camus. Au lieu de les identifier comme tels et de les transposer comme il sied, les traducteurs ont souvent tendance à en ignorer sciemment ou inconsciemment l'origine arabe et à les reproduire en hébreu à travers le prisme déformant du français. C'est ainsi que dans «Retour à Tipasa», le mot «Sahel » (de l'arabe sâhil) qui désigne les collines littorales est maladroitement rendu par סַ dans la traduction d'Ilana $\mathrm{H}$ abermann et presque aussi faussement par סטאה dans la version d'Aviva Barak. Cette dernière a tout de même jugé bon d'indiquer la longueur vocalique de la première syllabe en utilisant une mater lectionis $x$, mais elle ne s'est pas avisée du fait que le graphème - $h$ - du français reproduisait un /h/ pharyngal dont le $n$ hébreu serait une bien meilleure translittération. Quant à la voyelle finale, c'est un /i/ et non un /e/ ou un /a/. En reconstituant une voyelle /a/ au lieu du /i/, Aviva Barak s'est laissée piéger par un phénomène de graphie inverse. II semble qu'elle ait pris le e de Sahel pour la graphie francisée du phénomène phonétique de l'imalah (passage de /a/ à /ä/) ${ }^{22}$ qui caractérise l'arabe maghrébin (cf. l'arabe oriental wadi en regard de l'arabe maghrébin wäd francisé en oued). Elle a donc pensé bien faire en remontant la filière en sens inverse et en remplaçant /e/ par /a/, lors même qu'il représente un /i/ dans l'étymon arabe.

Le même genre d'interférence du français dans le passage de l'arabe à l'hébreu s'observe dans la traduction de L'exil et le royaume ${ }^{23}$. Dans «L'hôte» figure le toponyme arabe francisé El Ameur. Dans la pratique coloniale française, le digramme eu transposait le sukun arabe en fin demot. En somme, le phonème/ö/ était introduit comme une sorte de voyelle d'appui. Or le traducteur Zvi Arad a rendu El Ameur, c'est-à-dire Al-Amr, par אל אמֶ, sous prétexte que le /e/ hébreu sert normalement d'équivalent au phonème/ö/ dans la translittération du français. II aurait bien mieux valu transposer El Ameur par אל אמ̣ר. Au lieu de cela le traducteur est tombé dans le 
piège de la graphie inverse en transposant le digramme eu par un ségol lors même que le digramme eu est en fait l'équivalent d'un sukun.

II n'est pas rationnel de passer par le français pour transposer un mot arabe en hébreu. Ce détour par le français révèle que la langue arabe, pourtant apparentée génétiquement à I'hébreu et jouissant en outre du statut de langue officielle en Israël, est trop facilement évacuée des références culturelles israéliennes, au point que les traducteurs sont incapables d'identifier le mot arabe qui se dissimule derrière tel ou tel toponyme francisé.

Dela même façon, le lieu dit «rue du Fondouk » qui figure au début du paragraphe intitulé «Les Jeux » ( «Le M inotaure ou la halte d'Oran », L'été) est translittéré en hébreu d'après la prononciation française: רחוב די פונדוק. Outre cette bévue dans la translittération du vieux mot grec $\pi \alpha v \delta$ okcliov passé à l'araméen (פונדק) puis à l'arabe (funduq), on remarquera la transposition machinale de la préposition du par T, comme si ce mot-outil était solidaire de Fondouk ou comme si Fondouk était aussi français que du.

Cette incapacité à reconnaître avec exactitude l'arabité de certaines notes de couleur locale pied-noir relève vraisemblablement d'une sorte d'a priori qui consiste à penser que dans un texte francais tout est français, même quand ce français laisse transparaître des réalités nord-africaines très superficiellement francisées, lesquelles gagneraient à être replacées dans leur contexte arabe originaire.

Inversement, l'expression «bleus de chauffe» qu'Aaron Amir avait traduite par a été indûment réorientalisée par Ilana Habermann qui a rendu ce terme désignant un habit tout ce qu'il y a plus occidental par סרבלי עבודה כחולים. Ces difficultés dans la transposition de la réalité algérienne d'avant l'indépendance tiennent en partie au fait que les Israéliens ont du mal à cerner le statut de cette expérience historique de la présence française séculaire en Algérie. Les processus d'acculturation qui ont abouti à l'intégration d'un grand nombre de mots arabes ou sabirs dans le parler pied-noir, puis dans le français métropolitain et inversement à la francisation progressive des masses musulmanes des deux côtés de la M éditerranée, représentent quelque chose d'à la fois étrange et familier : étrange, parce que la France est perçue comme un pays tout ce qu'il y a de plus européen et qu'il est difficile de comprendre que jusqu'en 1962, trois de ses départements se situaient en terre africaine; familier, puisque ces processus d'acculturation entre une population européenne ou occidentalisée et un substrat arabe ont également été à l'œuvre dans la constitution de l'identité israélienne actuelle. Mais comme l'analogie entre l'Algérie francaise et l'État d'Israël ne peut être menée jusqu'au bout et que les deux situations historiques sont irréductibles l'une à l'autre, en dépit de telle ou telle analogie structurelle ou superficielle pointant de-ci delà, il se crée un décalage et une source de contresens pour les traducteurs de Camus qui méconnaissent l'ancrage arabe de tel ou tel toponyme ou qui tiennent absolument à habiller des manœuvres arabes d'un saroual dont ils n'ont que faire.

Et puis n'oublions pas que I'horizon arabe auquel sont accoutumés les I sraéliens est celui du Mashreq, si différent à bien des égards de l'Occident arabe. L'opposition entre le Maghreb et le Mashreq vient donc se combiner avec le décalage Nord-Sud pour achever de brouiller certains repères dans le processus de transposition de Camus du francais à I'hébreu. La seule exception qui vient nuancer cette opposition 
entre Mashreq et M aghreb est la présence d'une importante communauté juive marocaine en I sraël. II n'est donc pas fortuit qu'une traductrice comme Aviva Barak qui est tombée dans certains pièges lorsqu'il sagissait de traduire des mots arabes francisés a fort bien su retranscrire en revanche le nom de famille juif marocain Amar en עמאר (nom du boxeur dans «Le Minotaure ou la halte d'Oran», L'été).

Enfin, il convient de noter que le terme «Mauresque» (L'étranger I,3) a subi des avatars intéressants selon les diverses traductions de L'étranger. Cet ethnonyme qui désignait les Arabes maghrébins dans le contexte de la colonisation française a une longue histoire: il semble refléter l'usage espagnol qui consistait à appeler Moros les Arabes ou les Berbères d'Andalousie et d'Afrique du Nord. Dans les traductions al lemande et américaine de L'étranger, ce terme très connoté est traduit par $M$ aurin et par Moor. Ce faisant, il se sont contentés de trouver dans leurs langues respectives l'équivalent étymologique du signifiant M auresque / M ora sans trop se poser de questions sur le signifié ou le référent de cet ethnonyme dans le contexte particulier de I'Algérie d'avant l'indépendance. Le premier traducteur israélien, Aaron Amir, a fort bien saisi la valeur exacte du signifié M auresque et il l'a rendu à l'ai de d'une périphrase précise, ערביה מבנות המקום, la mention מבנות המקום spécifiant qu'il s'agit d'une Arabe nord-africaine. Quant à la seconde traductrice, I lana H abermann, elle a fait l'économie de cette précision et elle a transposé «Mauresque» par ערביה, en partant du principe que mutatis mutandis, les deux termes sont équivalents du point de vue de leur charge référentielle. Curieusement, Aaron Amir, qui avait fort bien traduit «M auresque» dans la première version de L'étranger, a commis un faux sens dans sa traduction de La mort heureuse (II,3), puisqu'il transpose cet ethnonyme par ברברית. Dans le contexte de l'Algérie française et dans les usages de langage pieds-noirs, I'adjectif maure / mauresque ne désigne pas spécialement les Berbères, mais tous les Musulmans d'Algérie, qu'ils soient arabes ou kabyles.

À titre de comparaison, on peut également examiner la façon dont l'adjectif «maure» a été traduit, notamment dans l'expression toute faite «café maure». Zvi Arad, soucieux qu'il était de donner un équivalent dynamique de l'expression, avait traduit «café maure» par בתי הקפה של הילידים (dans L'exil et le royaume, «La femme adultère»). Près de trente-huit ans plus tard, Adina Kaplan a rendu ce même syntagme nominal par קפה מאורי (dans L'envers et l'endroit, «Entre oui et non»). Cette différence reflète une évolution dans les pratiques de la traduction qui ont cours en Israël : dans les années 1950 prévalait un style de traduction communicatif qui tenait surtout compte des impératifs de la langue-cible. De nos jours, on constate une tendance à la traduction informative et sémantique: I'expression קפה מאורי est tellement fidèle au texte-source qu'elle reproduit purement et simplement le signifiant français. Zvi Arad, en revanche, s'est efforcé de traduire le signifié de la même expression, comme s'il ne faisait pas confiance au discernement du lecteur.

Ainsi donc les traductions de Camus en hébreu ont fait apparaître un progrès de la littéralité aux dépens de l'effort d'adaptation. La traduction de La peste par Yonathan Ratosh en 1953 représente le cas extrême de cette volonté d'hébraïser le texte de Camus, tandis que la traduction toute récente de L'envers et l'endroit laisse au texte quelque chose de sa francité exotique. Entre ces deux pôles, les traductions de Zvi Arad représentent un assouplissement vis-à-vis de la rigidité stylistique d'un Ratosh, 
mais le traducteur de La chute est encore très soucieux d'acclimater le texte-source et de le rendre compatible avec la langue-cible. Ilana Habermann correspond à un tournant, car sa traduction de L'étranger se veut plus fidèle tout en évitant le mot-àmot. Enfin, les traductions d'Aviva Barak et d'Adina Kaplan nous mènent résolument dans le domaine de la traduction informative et sémantique.

Outre l'évolution du style littéraire dont il a été question ci-dessus, on peut imputer cette préférence nouvelle pour le littéralisme à la perception de la languecible par ses propres locuteurs. Tant que l'hébreu jouissait du respect dû à une langue classique, la fidélité au texte-source était sacrifiée à la vénération vouée à la languecible. À ce propos il n'est pas fortuit que l'âge d'or des belles infidèles ait correspondu à l'époque des classicismes français. $M$ ais à partir du moment où une génération nouvelle a remis en question ce respect jusqu'alors inconditionnel lors même qu'il s'exprimait de façon purement laïque, il a semblé moins important de respecter les conventions de la langue-cible que de reproduire très exactement la formulation de la langue-source. Cette évolution correspond aussi à la fin de l'époque des poètestraducteurs qui marqua longtemps la vie littéraire israélienne ${ }^{24}$. Pendant des décennies, les grands poètes étaient aussi des traducteurs, ce qui donna lieu à un goût pour les belles infidèles et à une influence de la praxis de traduction sur la création littéraire elle-même. À l'exception d'Aaron Amir, les traducteurs récents de Camus que nous avons étudiés ici sont avant tout des traducteurs: mais ce que la traduction gagne en professionnalisme, elle le perd en qualité littéraire. S'agissant d'un auteur aussi soigneux de son style que l'était Camus, ce littéralisme parfois poussé à l'extrême représente peut-être une trahison encore plus grave que ne l'étaient les belles infidèles d'un Yonathan Ratosh ou d'un Aaron Amir.

\section{NOTES}

1. Ce dépouillement viserait en définitive à un silence complet. Sur cette tension vers un silence idéal, voir l'analyse freudienne de l'œuvre de Camus par Costes (1973).

2.

3. שם, עמי 20.

4.

5לבר קאמי, הזר, תרג' אהרן אמיר, עם עובד-מסדה, תשכ"ז.

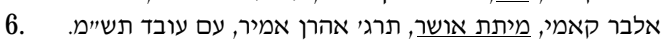

7. Voir Camus (1946).

8. Sur l'histoire complexe du mot grue en français, voir Wartburg (1952: 296-297); le sens technique de grue attesté dès le début du xii ie siècle semble être un calque du moyen néerlandais: voir Verdam, (1932: 311).

9.

אלבר קאמי כלולות / הקיץ, תרג' מאת אביבה ברק, מועדון קוראי מעריב, תל אביב תשמחי"ח.

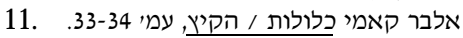

12. Voir Johann Wolfgang Goethe, West-Östlicher Divan (§ Übersetzungen) in Goethe, Werke I (Gedichte und Epen II), éd. E. Trunz, Munich: C.H. Beck, p. 255-258; trad. fr. par Henri Lichtenbeger, Aubier-M ontaigne, 1940. Ce passage a été commenté de façon lumineuse par Rolf Kloepfer (1967: 49-50).

אלבר קאמי, פנים וחוץ, תרג' עדינה קפלן, רשפים, תשניוז.

אלבר קאמי, מכתבים לידיד גרמני, תרג' עדינה קפלן, כרמל תשניוניו.

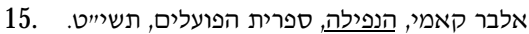

16. Paru sous le titre as-saqtah, Beyrouth, 1974.

17. Sur cette opposition entre les subjonctifs imparfaits en -ra et en -se, voir Molho (1975: 331-359); Hernández Alonso (1986: 297-298).

18. Elle remonte à Cicéron au moins. Voir Nida (1964: 11-29) et Kloepfer (1967: 21-27). 
19. Voir The Oxford English Dictionary, VI, p. 440 (s.v. geneva) et p. 517-518 (s.v. gin).

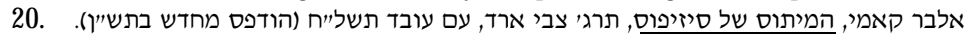

21. Rappelons que selon la définition donnée par le Trésor de la langue française, Gallimard, 1986, XII, 851 , s.v., le panama est «un chapeau d'été pour homme, en pailletrès fine, souple et léger, tressé avec de minces lanières découpées dans les feuilles d'un latanier d'Amérique centrale».

22. Pour une description détaillée de ce changement phonétique, voir Marçais (1903: 35-39).

23. Paru en appendice de la traduction de La chute mentionnée ci-dessus à la note 15.

34. Sur le reflet de cette évolution dans un autre domaine linguistique, voir Aslanov (1997).

\section{RÉFÉRENCES}

Asl anov, Cyril (1997): "Les voies de la traduction des oeuvres de l'Antiquité, classique en hébreu, comparaison de quelques traductions de textes poétiques», Les études dassiques, 65, p. $193-210$.

Bar th es, Roland (1972) : Le dégré zéro de l'écriture, Paris, Seuil.

Camus, Albert (1972): A Happy Death, tr. Richard Howard, New York, Alfred A. Knopf

(1957a) : The Fall, tr. Justin O'Brien, Londres, Hamish Hamilton.

(1957b) : Der Fall, tr. Guido G. Meister, Hambourg, Rowohlt.

(1956) : La Caida, tr. inconnu, Mexico.

(1955): The M yth of Sisyphus and Other Essays, tr. Justin O'Brien, New York, Alfred A. Knopf

( $2^{\mathrm{e}}$ éd. New York, Vintage, 1959)

(1981) : Der Fremde, tr. Georg Goyert et Hans Georg Brenner, Hambourg, Rowohlt.

(1946) : The Stranger, tr. Stuart Gilbert, New York, Alfred A. Knopf

(1948) : The Plague, tr. Stuart Gilbert, Londres, Hamish Hamilton.

Cost es, Alain (1973): Albert Camus ou la parole manquante, Paris, Payot.

Goethe, Johann Wolfgang (?): West-Östlicher Divan (Ü berstzungen), in Werke I (Gedichte une

Epen II), éd. de E. Trunz, Munich, C. H. Beck, p. 255-258.

(1940) : Le divan, tr. Henri Lichtenberger, Paris, Aubier-Montaigne.

H er nandez Al onso, César (1986) : Gramatíca funcional des Español, Madrid, Gredos.

KI oepf er, Rolf (1967): Die Théorie der literarischen Ü berselzung, Munich, Wilhelm Fink.

M ar çais, William (1903) : Le dialecte arabe parlé à Tlemcen, Paris, Leroux.

M ol ho, M aurice (1975): Sistématica des verbo español tome 1, M adrid, Gredos.

N ew mark, Peter (1972): Approaches to Translation, Oxford/N ew York, Pergamon Press.

$\mathrm{N}$ ida, Eugene A. (1964) : Towards a Science of Translating, Leyde, Brill.

The Oxford English Dictionary, Oxford, Clarendon Press 1989.

Trésor de la langue française, Paris, Gallimard, 1986.

Ver dam, J. (1932) : M iddelnederlandsch Handwoordenboek, La Haye, Martinus Nijhoff.

War t bur g, Walter von (1952): Französiches Etymologiches Wörterbuch, Bâle. 